\title{
Implementación de un Sistema Híbrido Tipo Trabajo Constante en Progreso (CONWIP) para Control de Producción en una Industria de Implementos Agrícolas
}

\author{
Anderson Hoose ${ }^{(1)}$, Luiz A. Consalter ${ }^{(1)}$ y Orlando M. Durán ${ }^{(2)}$ \\ (1) Programa de Pós Graduação em Engenharia Mecânica, Universidade de Passo Fundo, Bairro São \\ José, BR-285, CEP 99052-900, Cx Postal 611, Passo Fundo, Rio Grande do Sul-Brasil \\ (e-mail: andersonhoose@upf.br; lac@upf.br) \\ (2) Pontificia Universidad Católica de Valparaíso Ingeniería Mecánica. Avda. Los Carrera 01567, Quilpué, \\ Chile (e-mail: orlando.duran@ucv.cl)
}

Recibido May. 8, 2015; Aceptado Jul. 8, 2015; Versión final Oct. 6, 2015, Publicado Abr. 2016

\begin{abstract}
Resumen
Este trabajo tiene como objetivo presentar la aplicación práctica de un sistema híbrido de control de producción para la fabricación de piezas en la industria de maquinaria agrícola. En la propuesta, la sección de planificación de la producción emite una orden de fabricación y la entrega a fábrica donde se realiza la primera operación a la pieza. Una vez realizada la implantación del sistema hibrido de planificación de la producción se estudiaron sus efectos durante siete meses correlativos. Luego esos resultados fueron comparados con los siete meses inmediatamente anteriores a los de la implantación. Se pudo observar un aumento en el número de piezas producidas y vendidas. También se observó mejoras en la oferta de líneas de soldadura y una mejor explotación de las máquinas que sufren restricciones en cuanto a la falta de materia prima y reducción en los cuellos de botella de producción. Como conclusión se puede destacar la mejoría en la eficiencia de la línea en función de la sincronización entre la demanda y la producción.
\end{abstract}

\section{Implementation of a Hybrid System of the type Constant Work In Progress (CONWIP) for the Production Control in a Manufacturing Plant of Agriculture Machine Parts}

\begin{abstract}
This study addresses the practical application of a Hybrid System of Production Control in the manufacturing process of machine parts in an industry of agricultural machine part industry. In the proposal, the production planning section send and work order to the manufacturing section where the first step in the production chain starts. Once the hybrid system for the production control was implemented, its effects were registered and analyzed during the next seven consecutive months. The results were compared with the seven months just before the date of implementation. It was noticed after the study that there was an increase in the number of parts produced and sold. It was also observed that there was better supply of the weld lines and higher utilization of those machines that face restrictions regarding to the lack of raw material, quality problems, and increments of requests and a reduction of bottlenecks in the production lines. As a main conclusion it can be highlighted the enhancement of the line efficiency in terms of the synchronization between demand and production levels.
\end{abstract}

Keywords: production control; work in process; machine manufacturing; CONWIP 


\section{INTRODUCCIÓN}

La importancia de la industria de maquinaria agrícola es vital para la competitividad en los agronegocios. Hoy en día este sector de la industria está inserta en una economía globalizada y cada vez más competitiva (García; Silva, 2012). Los sistemas productivos en este sector de la industria no están exentos de los tradicionales problemas de gestión de sus operaciones. Alza en los stocks, altos tiempos de espera y una gran variedad en los productos llevan a la búsqueda por soluciones en el ámbito de la Planificación y Control de la Producción. Es en este ámbito es que surgen filosofías y técnicas para tratar de mejorar la gestión de los procesos productivos. Mula et al (2006) concluyen que existe la necesidad de nuevos sistemas para la planificación y control de la producción que simultáneamente consideren tanto la planificación de los materiales como de sus capacidades. CONWIP constituye uno de estos sistemas y se alza como una alternativa a los sistemas basados en la producción empujada y aquellos basados en tarjetas KANBAN.

Como Tubino (2000) y Moura (1989) señalan, los procesos de producción con múltiples etapas se pueden clasificar en dos tipos: la producción de empujar y de traccionar. Empujar producción significa elaborar periódicamente un programa completo de producción para cumplir con el Plan Maestro de Producción (PMP), desde la compra de materias primas hasta el montaje del producto terminado. Aun, hoy en día la mayoría de los sistemas tradicionales de producción de control emplea este sistema. Traccionar la producción significa no producir hasta que el cliente (interno o externo) de un proceso solicite la producción de determinado artículo generando el accionamiento de toda la cadena productiva a partir de la detección de satisfacer ciertos niveles de demanda. Según Guild (2013) y Barcos y Villela (2008), el sistema empujado de producción está controlado por un sistema central que considera las futuras previsiones de las etapas de la demanda y de los programas de producción. Correa (2001) argumenta que si la empresa tiene malas predicciones de demanda, las decisiones adoptadas sobre el funcionamiento del sistema de control también serán malas. En sistemas de producción controlados de esta manera, el flujo de material tiene la misma dirección del flujo de información cuando la liberación del material se produce a través del sistema.

Sistemas bien conocidos son el MRP (Material de Requirement Planning) y MRP II (Manufacturing Resources Planning), que proporcionan herramientas administrativas para planificar y controlar las actividades de fabricación con los objetivos a cumplir con los plazos de entrega con un mínimo de inventario. El sistema MRP indica cuánto y cuándo producir y adquirir los diversos componentes y materias primas, y el MRP II complementariamente proporciona la información para llevar a cabo el plan de producción que se sugiere en el PMP.

Dentro del sistema de empujar la producción con el fin de cumplir con el PMP, las actividades de la programación de la producción, como señala Tubino (2000), se dividen en tres grupos: la gestión de inventarios; el secuenciado de los trabajos (scheduling) y la de emisión y liberación de órdenes de producción y/o montaje. Sin embargo, Rivero (2006) y Moura (1989) aseguran que el MRP se utiliza para planificar artículos de demanda dependiente. Además, MRP requiere información de entrada que presente la demanda individual de los productos finales, teniendo en cuenta los plazos de entrega y de producción (tiempo de espera) el flujo del tiempo en cada etapa de la producción y el cálculo de los tiempos. De esta manera, las órdenes de producción y de compra se generan y se gestionan en el sistema.

En el nivel de planificación, y de acuerdo a Mula et al. 2005, en el MRP todos los cálculos de requerimientos se basan sobre un Plan Maestro de Producción, que a su vez se deriva de previsiones de demanda o pedidos de clientes, sin embargo, éste no es el caso del nivel operativo, donde MRP es claramente un sistema empujado. Así, los programas generados por el MRP provocan el inicio de los procesos de producción para satisfacer las fechas de entrega. Las piezas requeridas se procesan y pasan a la siguiente etapa hasta que se produce el montaje final.

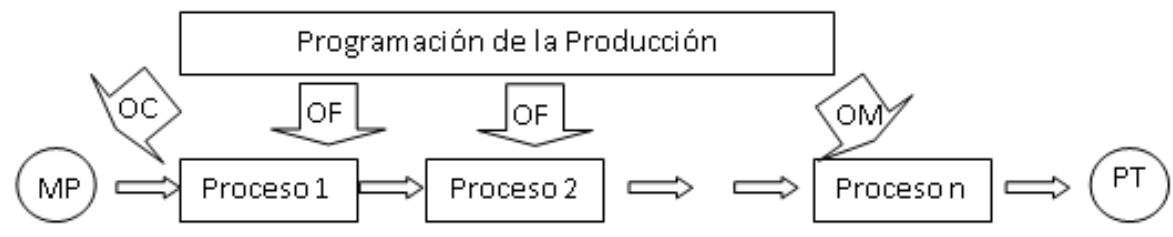

Fig. 1: Flujo de Programación de la Producción Convencional (Tubino,2000)

La figura 1 muestra la perspectiva de la programación convencional, es decir, la producción empujada. Aquí las órdenes de compra (OC) son emitidas para la adquisición de la materia prima (MP), la emisión de órdenes de fabricación (OF) en el caso intermediario y de montaje (OM), llegando a obtención del producto terminado (PT), (Tubino, 2000). 
En el sistema de producción traccionada hay una cierta cantidad de material en stock en cada etapa de la producción, en un proceso posterior (cliente interno o externo) se puede solicitar la producción de un artículo sólo en la medida y momento que se han consumido artículos existentes en esos stock intermediarios (Kootanaee, 2013; Caro, 2012; Tubino, 2000 y Moura, 1989). En este caso, el programa de producción utiliza la información del Plan Maestro de Producción para la emisión de las órdenes de producción sólo en la última etapa del proceso de producción, es importante tener en cuenta que "Just-inTime" (JIT) y Kanban no son sinónimos. JIT es una filosofía completa, mientras Kanban es una técnica para "traccionar" que se utiliza en el contexto más amplio de esta filosofía, con el fin de hacer la programación y control de las actividades de producción rápida y sencilla, evitando totalmente la sobreproducción y eliminando los inventarios extras y excesivos controles en la producción.

En japonés Kanban significa "Tarjeta" y en ella normalmente se registran tres datos básicos: (Qué) el nombre o número de pieza a producir, (Cuánto) el nivel de la demanda proveniente de la etapa posterior y (Cuándo) instante o fecha en que se van a procesar dichas piezas. Así, el JIT, considera que las piezas no lleguen antes de que se necesiten y sólo cuando sean necesarias (Sucena, 2013; Neto, 1992 y Ohno, 1997). Por lo tanto, en la producción del sistema traccionado, Kanban, no se produce nada hasta que el cliente (interno o externo) haga la solicitud concreta. En este caso, y como ya fue comentado, las órdenes de fabricación se emiten sólo para el montaje final basándose en la PMP (Figura 2) (Alves y Santos, 2013; Domingo et al. 2004 y Moura, 1989). Así, el sistema Kanban simplifica en gran medida las actividades a corto plazo realizadas por la Planificación de la Producción y Control de procesos por lotes repetitivos (Gonzalez et al., 2004). Son funciones del sistema Kanban:

1. Minimizar el inventario en proceso, evitando que el exceso de producción, a través de la definición de qué, cuánto y cuándo producir;

2. Simplificar el proceso de control de inventario;

3. Reducir o acelerar el tiempo de ejecución de la producción;

4. Prevenir la transmisión de las fluctuaciones de la producción;

5. Elevar el nivel de control visual de la planta, proporcionando información sobre el ritmo de producción y del transporte entre etapas productivas

6. Reaccionar más rápidamente a los cambios en la demanda;

7. Reducir los defectos, evitando la evolución a lo largo de la línea de productos defectuosos a través del proceso productivo.

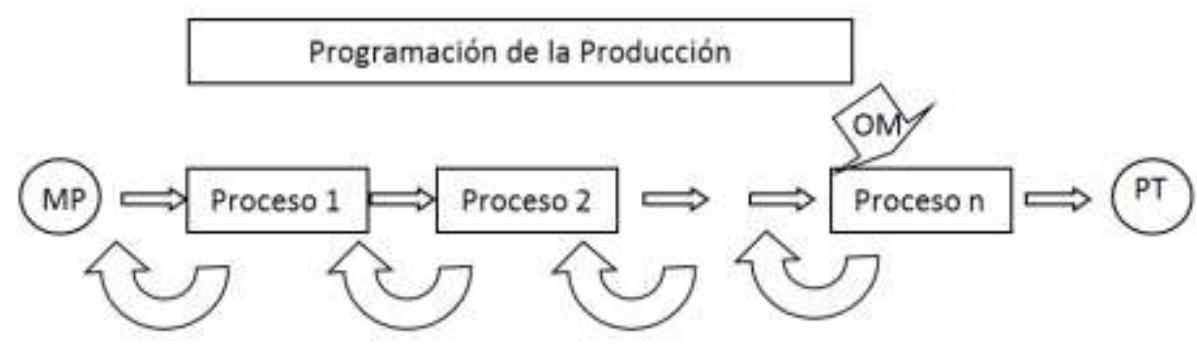

Fig. 2: Flujo del Sistema traccionado de producción (Tubino, 2000)

De acuerdo a Martins y Laugeni (2005), los sistemas de control de la producción, traccionado y empujado, no son mutuamente excluyentes. Estos pueden coexistir, ya que hay diferencias significativas entre ellos, por lo tanto su aplicabilidad depende de la organización de la fábrica y características de la demanda de los clientes. CONWIP (Constant Work In Process), es un sistema híbrido de control de la producción que comparte los beneficios de la reducción de inventario con Kanban en una suerte de configuración híbrida. Como de Barcos y Villela (2008) y Spearman (1990) demuestran CONWIP es aplicable a un mayor número de entornos productivos, ya que funciona tanto con las decisiones basadas en el nivel de inventario (traccionar la producción), como sobre la base de las transformaciones de las necesidades del Plan Maestro de producción en necesidades de artículos (producción empujada). Según Souza (2011), Barco y Villela (2008) y Rivero (2006), CONWIP es una estrategia de producción que define un nivel máximo de inventario en proceso, a lo largo de la línea de producción, o dentro de un sistema. Así, para Khojasteh-Ghamari y Sato (2012), la superioridad de CONWIP en relación al Kanban está en los menores stocks en proceso y en la menor cantidad de tarjetas para su control. Utilizando el CONWIP se asegura una nivelación de la producción, y se permite producir determinado artículo guiado por la demanda, con la premisa del sistema traccionado con un control mínimo de inventario, utilizando el MRP sólo como un sistema de información y no como un sistema de control de producción. Últimamente se han propuesto diversas versiones del 
CONWIP. Prakash y Chin (2015) presentan un estudio donde describen y clasifican 15 variantes de este sistema. Una de las preocupaciones en aplicaciones de CONWIP apunta a la determinación de la cantidad de tarjetas (y por lo tanto al tamaño de los stocks en proceso). En este sentido, Huang et al. (2015) proponen el uso de simulación para dimensionar estos parámetros en una línea de ensamblado que utiliza el enfoque CONWIP. Belisario y Pierreval (2015) proponen un enfoque de programación genética para apoyar la decisión sobre en qué circunstancias vale la pena modificar el número de tarjetas.

Al igual que el Kanban, el CONWIP puede trabajar con un recipiente o contenedor estándar (con una tarjeta fijada a él) que permite la el traslado, entre estaciones de trabajo, de cierta cantidad fija de material a procesar De esta manera se puede controlar el material que entra en el sistema al comienzo de la línea, pasando por todas las etapas del proceso de producción, hasta llegar al final del proceso productivo, constituyéndose en una forma específica de Kanban, basada en señales (Sultana y Ahmed, 2013; Souza, 2011; Barco y Villela, 2008 y Rivero, 2006). En este sistema hay un número preestablecido de tarjetas que define una cantidad fija y constante de trabajo en proceso para todo el sistema o línea productiva.

La Figura 3 muestra, esquemáticamente, el flujo de materiales y de información del CONWIP, que busca respetar una secuencia de tipo FIFO (First in First out), utilizando el sistema de control de producción traccionado (pull).

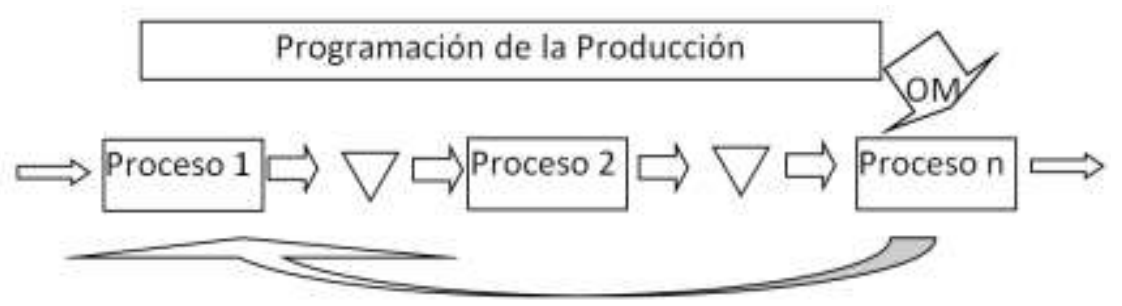

Fig. 3: Flujo de materiales y de información en CONWIP (Rivero, 2006)

\section{CASO DE APLICACIÓN}

Para mostrar la implantación de un sistema CONWIP se describe a continuación el caso de una empresa productiva de máquinas agrícolas localizada en el estado de Rio Grande do Sul, Brasil. La empresa cuenta con tres plantas, siendo la primera (planta A) la destinada al sector de Estamparía y que cuenta con máquinas de grandes dimensiones encargadas de las operaciones de corte por láser y por plasma, guillotinas, prensas y plegadoras. En la planta B se localiza el sector de mecanizado, preparado para operar en piezas de grandes dimensiones y en conjuntos soldados. Finalmente en la fábrica $\mathrm{C}$ se ubica el sector de soldadura. Ahí se ubican dos líneas llamadas de productos y accesorios respectivamente. En esta planta los puestos de trabajos son abastecidos por carros kit que contienen las piezas necesarias para cada una de las operaciones que conducen a la obtención de un producto o accesorio. De manera general, en las plantas A y B, la maquinaria y equipos de fábrica se agrupan de acuerdo a un diseño funcional (job shop), que se caracteriza por centros de trabajo separados, donde se procesa una variedad de piezas y se compone de agrupamientos de máquinas idénticas y similares, en los que se las piezas se van moviendo (en lotes) y donde hay normalmente grandes cantidades de trabajo en proceso.

La planificación y control de la producción (PCP) se hace de la siguiente manera: el departamento de PCP recibe los pedidos desde el área comercial y genera el Plan Maestro de Producción (PMP). Con el PMP se computan las necesidades de abastecimiento y, también se generan las órdenes de fabricación (OFs), que son enviadas a los sectores de estamparía y mecanizado. El PMP es calculado una vez al mes, siendo que las OFs son emitidas semanalmente. Posteriormente se considera una semana para la soldadura de componentes y una semana para el ensamblado final, o sea, un lead time de tres semanas, entre la emisión de las OFs hasta la conclusión del producto final. O sea, considerando el PMP, se fijan metas de producción para cada una de las fábricas, actuando bajo un sistema de gestión de producción empujada. A través del PMP, también se realiza el análisis de la capacidad de producción o balanceo de carga máquina, donde se consideran el total de horas programadas dentro del mes y la disponibilidad de horas máquina.

Inicialmente se realizó un análisis y diagnóstico de la situación actual y de los principales problemas de gestión de la producción existentes. Esto se logró a partir de entrevistas con el supervisor del sector de estamparía y con los líderes de las demás áreas involucradas en la fabricación de piezas, o sea, corte térmico y mecanizado. Además se hicieron entrevistas con los supervisores del departamento de PCP y logística interna. Se hicieron observaciones en terreno en un periodo que llevó alrededor de ocho meses. Basándose en todos estos antecedentes se pudo constatar los siguientes aspectos: 
1. Fallas en el secuenciado de las órdenes de fabricación;

2. Repetición de lotes de piezas iguales dentro de la misma semana con los consabidos costos (repetitivos) de set up en diversas máquinas;

3. Dificultad para visualizar los lotes de producción que deben ser fabricados en una determinada semana, toda vez que como consecuencia de los problemas encontrados en un dado instante se pueden encontrar lotes programados en hasta tres semanas diferentes;

4. Dificultad en la atención a pedidos urgentes (anticipaciones) o incremento de tamaños de lotes, no previstos en la programación semanal;

5. Acúmulo de gran cantidad de piezas en proceso, dificultando el establecimiento de prioridades conforme fechas de entrega de las OFs;

Cuanto a la planificación de la producción durante el período de análisis, fue programada un promedio de dieciséis planes de producción para la fabricación de piezas dentro de una misma semana. El problema se produce principalmente en el sector de la producción donde se realiza la operación de corte del material. Debido a que se espera una cierta cantidad de órdenes para acumular la fabricación de un solo calibre que sea suficiente para así hacer un mejor uso del set up de la máquina. Se puede observar que de esta manera, hay demoras en la entrega de las piezas hacia el sector de mecanizado.

\section{IMPLANTACIÓN DEL SISTEMA CONWIP}

Para la implantación del sistema propuesto, se consideró un total de 41 piezas que forman parte de 36 conjuntos finales. Los criterios utilizados para la selección de estas piezas fueron los siguientes: estas serían piezas mecanizadas a partir de barras redondas o cuadradas de acero laminado y cuya producción mensual no fuera menor a la capacidad del contenedor de transporte (lote mínimo). Una vez seleccionadas las piezas, se calculó y se produjo el stock suficiente para atender la demanda de dos meses por cada pieza, más un mes adicional, considerado el stock de seguridad (usando como dato el promedio de los últimos seis meses de unidades vendidas para cada pieza). El sistema híbrido de control de la producción se implementó usando dos tipos de tarjetas, uno verde y otro de color rojo (Figura 4). El tamaño del lote para las tarjetas verdes se considera de dos meses de demanda, mientras que el tamaño del lote en las tarjetas rojas es de un mes (stock de seguridad). Observe la figura 4, en ella se pueden apreciar cuatro instantes en el funcionamiento del sistema. Considerando una pieza hipotética cualquiera, el ciclo se inicia con el contenedor lleno, a medida que las unidades del stock comienzan a ser "consumidas por producción" se llega al nivel mínimo de stock, ahí se le fija al contenedor la tarjeta roja y la tarjeta verde pasa a cumplir el papel de orden fabricación por las piezas que están en el contenedor. Después de dos semanas (lead time de producción para estas piezas), la pieza es reabastecida al stock con la tarjeta verde nuevamente fijada al contenedor. Note que si durante esas dos semanas ocurre algún tipo de improviso, aun se posee un stock de seguridad equivalente a un mes de trabajo.

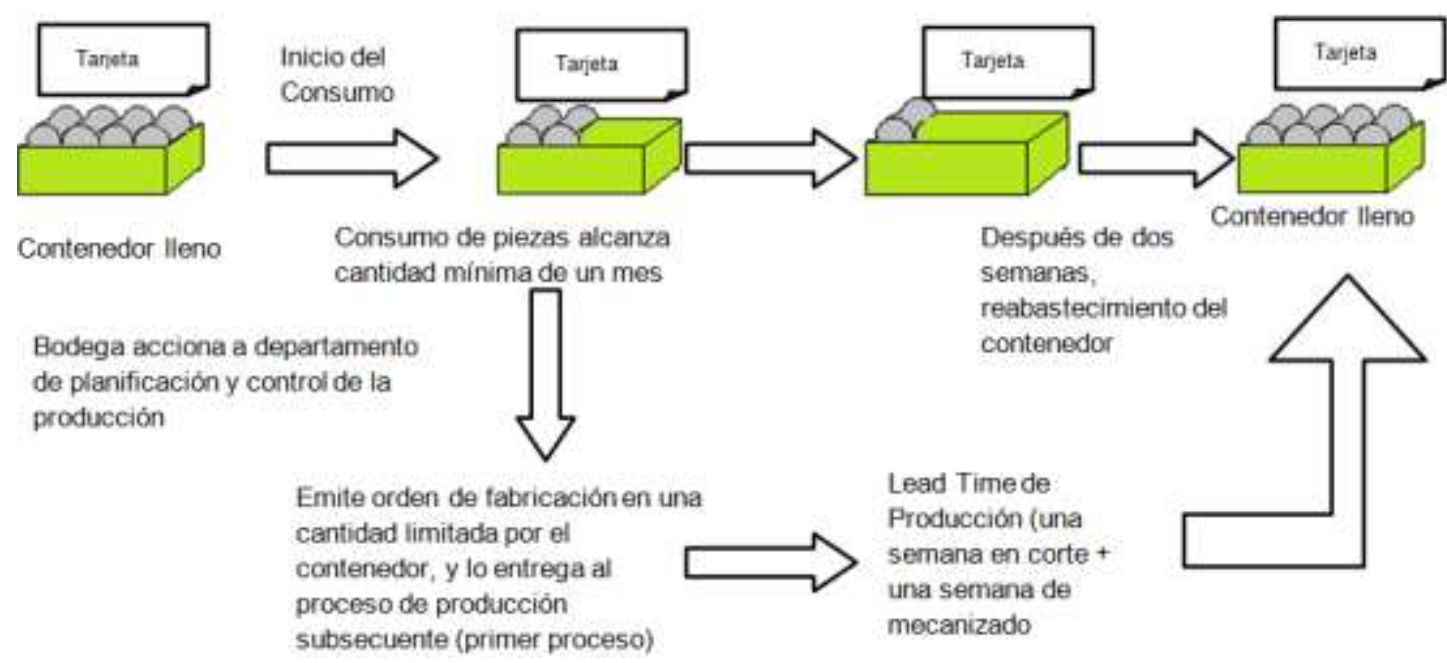

Fig. 4: Flujo de accionamiento y de reabastecimiento (CONWIP)

La operación con la tarjeta tiene solamente la finalidad de controlar el nivel mínimo de stock (punto de pedido), quedando a criterio de la PCP la emisión de la orden de fabricación con la cantidad necesaria de piezas para recomponer el stock. 
En la figura 5 se aprecia la dinámica de los niveles de stock y el consumo de la pieza mostrada aquí como ejemplo.

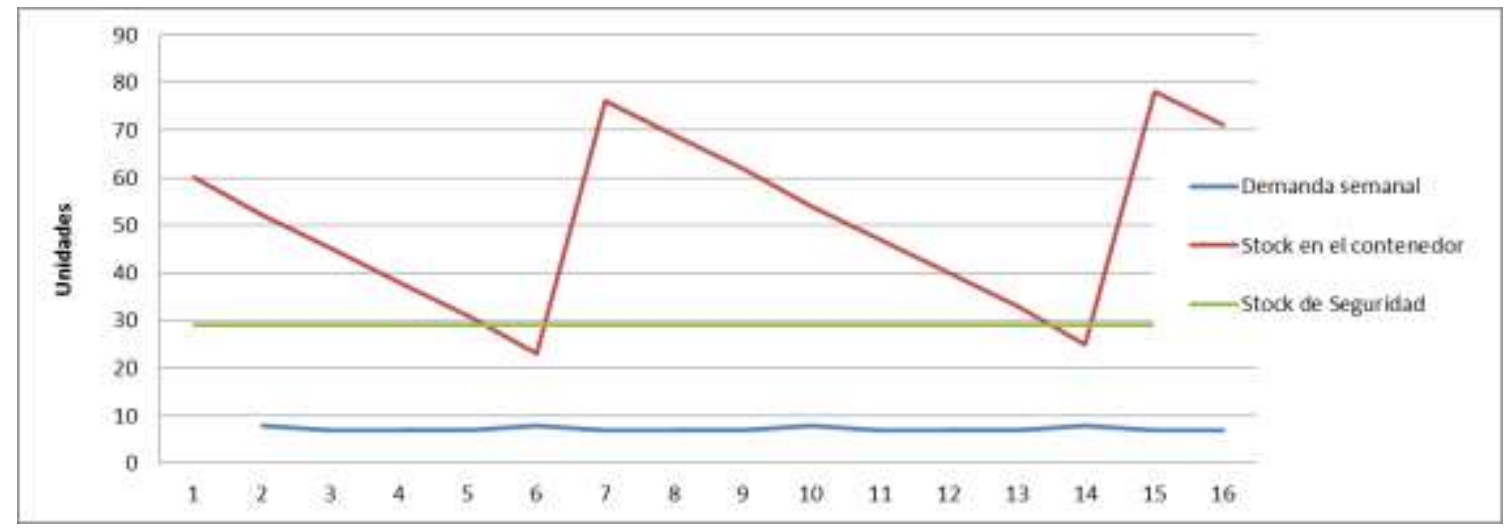

Fig. 5: Dinámica de los niveles de stock

La tabla 1 muestra los valores asociados a la dinámica ya comentada más arriba durante 15 semanas consecutivas.

Tabla 1. Movimiento de los stocks y de la producción con el sistema implementado.

\begin{tabular}{|c|c|c|c|c|c|c|c|c|c|c|c|c|c|c|c|c|}
\hline & Stock Inic. & Sem. 1 & Sem. 2 & Sem. 3 & Sem. 4 & Sem. 5 & Sem. 6 & Sem. 7 & Sem. 8 & Sem. 9 & \begin{tabular}{|l|} 
Sem. 10 \\
\end{tabular} & \begin{tabular}{|l|} 
Sem. 11 \\
\end{tabular} & \begin{tabular}{|l|} 
Sem. 12 \\
\end{tabular} & \begin{tabular}{|l|} 
Sem. 13 \\
\end{tabular} & Sem. 14 & Sem. 15 \\
\hline Demanda semanal & & 8 & 7 & 7 & 7 & 8 & 7 & 7 & 7 & 8 & 7 & 7 & 7 & 8 & 7 & 7 \\
\hline Stock en el contenedor & 60 & 52 & 45 & 38 & 31 & 23 & 76 & 69 & 62 & 54 & 47 & 40 & 33 & 25 & 78 & 71 \\
\hline Stock de Seguridad & 29 & 29 & 29 & 29 & 29 & 29 & 29 & 29 & 29 & 29 & 29 & 29 & 29 & 29 & 29 & 29 \\
\hline Acciona producción & & & & & 60 & & & & & & & & 60 & & & \\
\hline Conclusión del lote & & & & & & & 60 & & & & & & & & 60 & \\
\hline
\end{tabular}

\section{RESULTADOS Y DISCUSIÓN}

Una vez realizada la implantación del sistema hibrido de PCP se estudiaron sus efectos durante siete meses correlativos. Luego esos resultados fueron comparados con los siete meses inmediatamente anteriores a los de la implantación. Se puede considerar que esta comparación es adecuada y satisfactoria puesto que las condiciones de mercado y organizacionales al interior de la fábrica prácticamente no se vieron alteradas, con excepción del nuevo enfoque usado (CONWIP). Durante el análisis se produjeron 41 piezas diferentes en la línea de producción. Este tipo de comparación está basada en la aplicada por Harrod y Kanet (2012). Basándose en los niveles de stock en proceso y de la cantidad de tarjetas necesaria para realizar el control de producción.
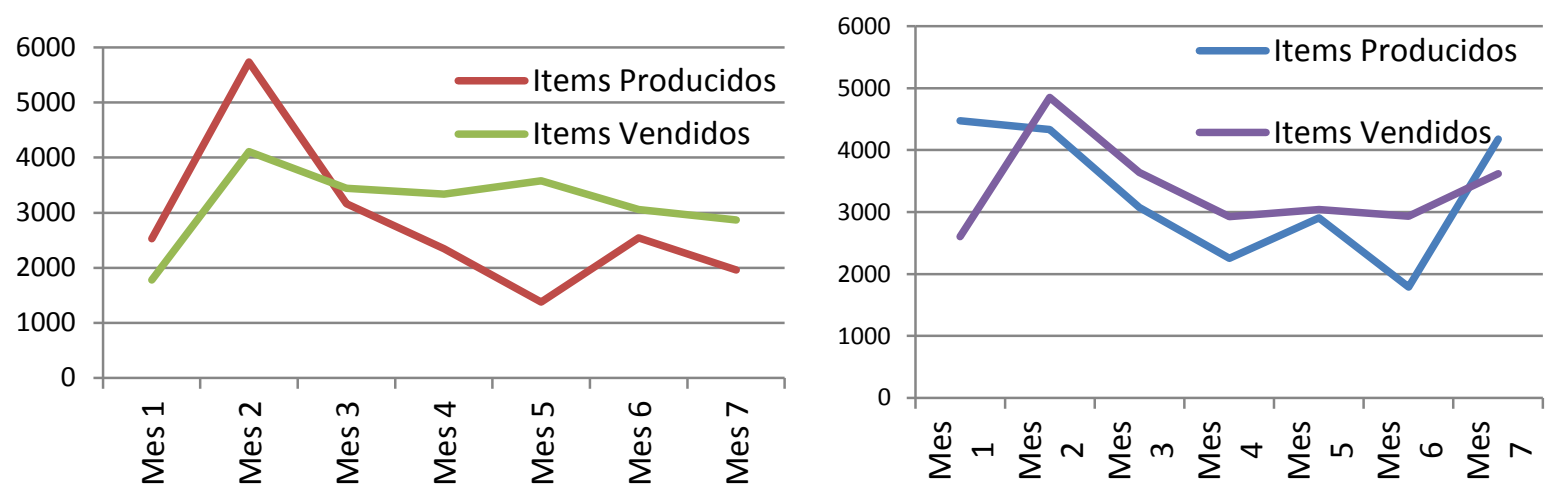

Fig. 6: Comparación entre Sistema Empujado(a) y CONWIP

Al realizar la comparación entre ambos períodos se pudo constatar que la media de la disparidad entre producción y ventas de los ítems con el sistema CONWIP fue inferior a la misma cuando se usaba el sistema tradicional. Esto resulta ser positivo, pues todo lo que se produce es vendido. Además demuestra una mejor sincronización entre las necesidades y la producción. En promedio, por el sistema tradicional hubo una disparidad entre los producido y vendido de $11,4 \%$ mientras que al implantar el sistema CONWIP esa diferencia correspondió al 2,6\%. 
De esta manera se puede considerar que usando el sistema CONWIP hay una mejor sintonía entre lo que se produce y lo que se vende. Esto puede apreciarse en la figura 6 . La disparidad inicial bajo el sistema CONWIP se debe a la generación de un stock de seguridad. Haciendo una comparación entre la cantidad de órdenes de fabricación por el sistema tradicional y el sistema CONWIP, se muestran los resultados en la figura 7 . Se puede apreciar una reducción de $15 \%$ en la cantidad de órdenes de fabricación completadas, lo que indica una menor cantidad de set ups en la línea para llevar a cabo una producción equivalente entre los meses comparados. El abastecimiento de las líneas de soldadura considerando el sistema tradicional y el sistema CONWIP se muestra en la figura 8. Los números representan la cantidad de ítems abastecidos a la línea por cada mes.

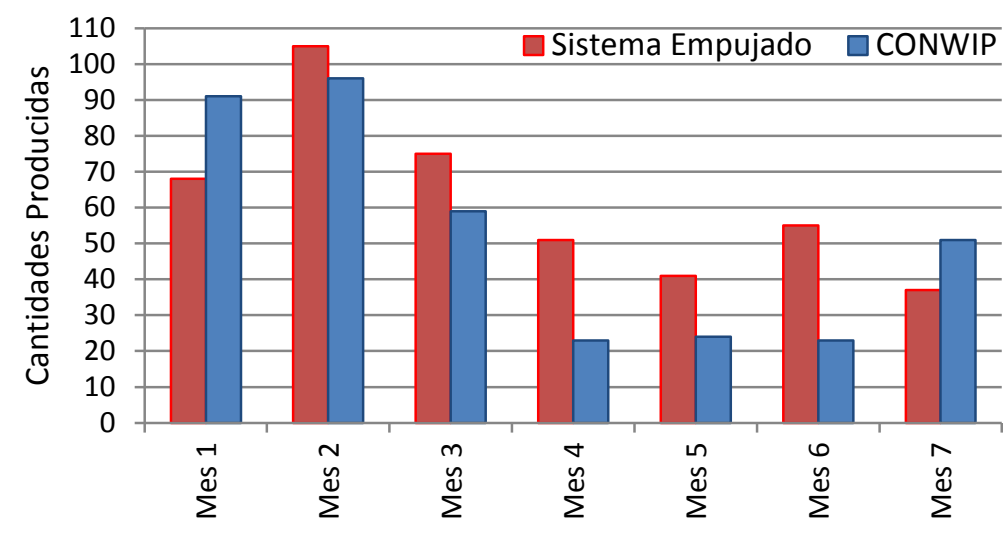

Fig. 7: Órdenes de Fabricación Producidas (Sistema Empujado vs. CONWIP)

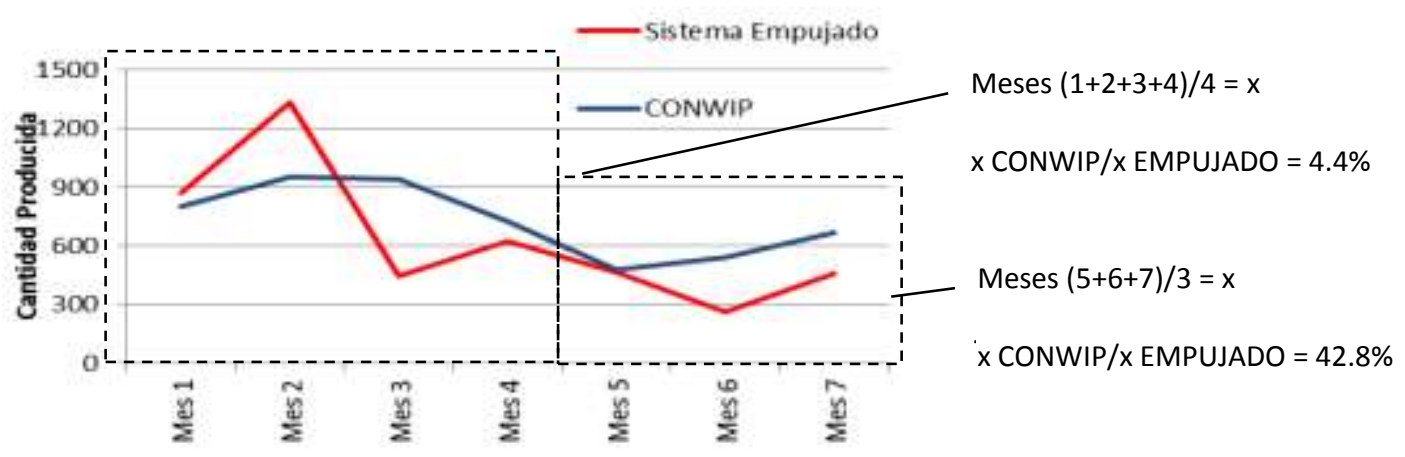

Fig. 8: Ítems abastecidos (Sistema Empujado x CONWIP)

Se puede observar que el período que presentó un mejor índice de abastecimiento fue el segundo mes, donde, usando el CONWIP se pudo abastecer una cantidad de 951 unidades a la línea de soldadura. Por otro lado, el menor índice de abastecimiento fue registrado en el sexto mes, donde se abastecieron 544 unidades usando el método propuesto e implantado. En la figura 8 se observa una menor oscilación en la cantidad de ítems abastecidos, haciendo que el ritmo de abastecimiento a la línea de soldadura sea más constante que con el sistema tradicional, además de ser menor en el caso del sistema empujado. Al comparar el nivel de abastecimiento provocado por ambos sistemas (actual y propuesto) se percibe que la diferencia asciende a un $14,6 \%$ a favor del sistema propuesto.

De los resultados obtenidos se pudo hacer las siguientes observaciones:

i) La formación de stocks mínimo posibilita la venta de determinados ítems evitando el desabastecimiento atendiendo de mejor manera la demanda;

ii) Como anteriormente los lotes de producción de los ítems eran bajos, la producción aguardaba en ciertos casos, la llegada de más de una orden de fabricación para iniciar el corte de piezas o su mecanizado para aprovechar de mejor manera el set up de las máquinas, lo que podía generar esperas en ciertos lotes y productos. Con el CONWIP hay solamente una orden de fabricación, evitando la emisión de más de una orden de producción para las mismas piezas; 
iii) Los cuatro primeros meses de implantación del CONWIP presentaron un promedio de desempeño de abastecimiento de la línea de soldadura con una diferencia de 4,4\% y para los últimos tres meses esta diferencia ascendió a $42,8 \%$ lo que hace pensar que existe un periodo de adaptación y suavizamiento al nuevo sistema después de su implantación; $y$

iv) En relación a los costos de implantación, estos pueden ser considerados bajos, pues en el caso de esta empresa ya se contaba con un espacio para la instalación en el almacenamiento de las áreas de acondicionamiento de los receptáculos (contenedores) en los que se basa físicamente el sistema.

\section{CONCLUSIONES}

Con la implantación de este sistema híbrido de control de la producción, y que tiene como objetivo la incorporación de dos formas de sistemas de control el "empujado" y el "traccionado", se logra iniciar la producción de sólo aquellos elementos que son necesarios para abastecer el stock final, que a su vez es consumido a través de la acción del cliente. La mejora y la cercanía entre lo que se vende y lo que se produce, se debe en gran parte a los stocks de seguridad, que sirven como amortiguadores de ineficiencias en los procesos de producción para la fabricación de piezas y mejorando el nivel de servicio a la demanda.

Se pudo observar como aspecto positivo a lo largo de este trabajo que la diferencia media entre lo que se produce y se vende varió de $11,4 \%$ a $2,6 \%$, lo que indica una reducción en la cantidad de trabajo en proceso, y refleja el aumento de artículos producidos y vendidos. Incremento medio del $14,6 \%$ en el suministro de líneas de soldadura y un mejor aprovechamiento del setup de la máquina o de la línea como un todo, con una reducción del $15 \%$ en la cantidad de órdenes de producción generadas (reducción del número de lotes ordenados).

Junto con esto, se revelaron algunos factores que afectan los trabajos e impiden mejores resultados, tales como: falta de materia prima, incremento de las solicitudes de emergencia y aumento del lead time debido a roturas de máquina, eventuales problemas de calidad y los retrasos en el mes anterior que se reflejan en el rendimiento del comienzo del mes siguiente.

\section{REFERENCIAS}

Alves, J.A. y A.P. Santos Logística Lean para Redução dos Efeitos da Variação da Demanda no Abastecimento de Linhas de Produção. Revista Perspectivas Contemporâneas, 8(1), 53-66 (2013)

Barco, C. F. y F. B. Villela. Análise dos Sistemas de Programação e Controle da Produção. XXVIII Encontro Nacional de Engenharia de Produção, Rio de Janeiro, (2008)

Belisario, L. y H.Pierreval. Using genetic programming and simulation to learn how to dynamically adapt the number of cards in reactive pull systems, Expert Systems with Applications, 42(6), 3129-3141,(2015)

Caro, H., Suero,D. y J. López. Comparing Kanban, CONWIP and Hybrid Pull Systems. Ingeniare, Universidad Libre-Barranquilla, 7(12), 99-105, (2012)

Correa, H.L., Nogueira Gianesi, I. G. y M. Caon. Planejamento, Programação e Controle da Produção. 4ª Edição. São Paulo: Editora Atlas S.A., (2001)

Domingo, R; Gonzalez, C y Calvo, R. Análisis del Diseño y Programación de Celdas en Entornos de Fabricación Ágil. Inf. tecnol. , 15(1) 55-60 (2004)

Garcia, B.P., Gründling, R. Dal Belo Leite, J.G., Schanberg Brandão, F. y T. Nunes da Silva. O Setor de Máquinas Agrícolas Brasileiro e o Comércio Internacional. http://online.unisc.br/seer/index.php/cepe/article/ view/ 652/435 Acceso: 03/11/2012

González-R, P.L., Framinan J. M. y H. Pierreval. Token-based pull production control system: an introductory overview. J Intell Manuf. 23 (1), 5-22 (2012)

Guild, D. MRP e Kanban. Together Again For The First Time! APICS San Antonio PDM, April 16, (2013).

Harrod, S. y J.J. Kanet. Applying work flow control in make-to-order job shops. Int. J. Production Economic, 143(2):620-626 (2012)

Kootanaee, A. J., K. N. Babu y H. F. Talari. Just-in-Time Manufacturing System: From Introduction to Implement. International Journal of Economics, Business and Finance. 1(2), 07-25, (2013) 
Martins, P.G. y F.P. Laugeni. Administração da Produção. 2ª Edição. São Paulo: Saraiva, (2005)

Moura, R. A. Kanban. A Simplicidade do Controle da Produção. São Paulo: Instituto de Movimentação e Armazenagem de Materiais, IMAM, (1989)

Mula, J., Poler, R. y J.P.Garcia. Evaluación de Sistemas para la Planificación y Control de la Producción. Inf. tecnol., 17(1) 19-34, (2006)

Neto, L. M., Sistema de Produção com Inventário Minimizado: abordagem técnico-financeira. São Paulo: Instituto de Movimentação e Armazenagem de Materiais, IMAM, (1992)

Ohno, T., O Sistema Toyota de Produção. Além da Produção em Larga Escala. Porto Alegre: Bookman, (1997)

Prakash, J.; Chin, J. F., Modified CONWIP systems: a review and classification, Production Planning \& Control, 26(4), 296-307. (2015)

Rivero, L. E. B., Motta E.R. y J.A.P.Rodriguez. CONWIP un Sistema de Control de producción. LACCEI International Latin American and Cariblean Conference for Engineering and Tecnology. Puerto Rico, (2006)

Sato, R., Khojasteh-Ghamari, Y., An integrated framework for card-based production control. J Intell Manuf June 2012, 23(3), pp 717-731 (2010)

Silva, L.C. da. Agronegócio e Organização de Cadeias Produtivas. II Semana Acadêmica de Eng. AgrícolaEngenharia do Agronegócio. www.agais.com/manuscript/ms0107_agronegocio.pdf. Acceso: 03/11/2012.

Souza, F. B. de, Rentes, Freitas A., y O.L.Agostinho. A interdependência entre sistemas de controle de produção e critérios de alocação de capacidades. Gestão \& Produção, 9(2), 215-234. (2002)

Spearman, M.L., Woodruff, D.L. y W.J.Hopp. CONWIP: a pull alternative to KANBAN. Int. J. Prod. Res., 28(5), 879-894, (1990)

Sucena, M., Engenharia de Produção. Planejamento e Controle da Produção. http://www.sucena.eng.br// eng_producao/UNESA_PCP_108_2013_1.pdf Acceso: 04/10/2014.

Sultana, I.y I. Ahmed. A state of art review on optimization techniques in just in time. Dept of Industrial and Production Engineering, Bangladesh Univ. of Eng. and Technology (BUET), Bangladesh. October (2013)

Tubino, D. F., Manual de Planejamento e Controle da Produção. $2^{\underline{a}}$ ed. São Paulo: Atlas, (2000) 
